\title{
Acquisition of a nose-poke response in rats as an operant
}

\author{
CHARLES W. SCHINDLER, ERIC B. THORNDIKE, and STEVEN R. GOLDBERG \\ NIDA Addiction Research Center, Baltimore, Maryland
}

(Vincent M. LoLordo, Sponsor)

\begin{abstract}
Acquisition of a nose-poke operant response was investigated in rats. The baseline level of this response was high and acquisition with food reinforcement occurred rapidly, particularly when compared with a leverpress operant response. A variety of control procedures clearly indicated that this response was acquired due to the contingency between nose pokes and food reinforcement. The response was also sensitive to manipulations of delay of reinforcement and fooddeprivation level. Acquisition was slowed with longer delays of reinforcement and with decreases in deprivation level. Therefore, the nose-poke response appears to be particularly useful for the study of the acquisition of operant responses.
\end{abstract}

The use of a nose-poke response as an operant has received relatively little attention in animal learning and behavior. In fact, most of the research on the nose poke has used either mice as subjects (e.g., Levine, Bornschein, \& Michaelson, 1977) or intracranial self-stimulation as a reinforcer (e.g., Mekarski, 1989). Despite this lack of investigation, the nose-poke response would appear to be useful for the study of the acquisition of operant responding in rodents. In naive rodents, nose-poke responses occur at a relatively high baseline rate-typically higher than that observed for leverpressing (Mekarski, 1989). Therefore, an animal would quickly come into contact with response contingencies, without intervention of the investigator. For example, leverpressing often requires shaping of the response by successive approximation, a procedure in which experimental conditions are difficult to control (e.g., Pear \& Legris, 1987). Therefore, the purpose of the present study was to investigate in detail the acquisition of the nose-poke response in rats.

During acquisition sessions, rats were placed into an operant chamber with two nose-poke operanda. One hole was defined as correct, and nose pokes to this hole were reinforced by food delivery. The other hole was defined as incorrect; responses to this hole had no scheduled consequence but served as an index of the baseline level of nose poking. If nose pokes did not occur, food pellets were delivered on a variable-time (VT) schedule, which also served as food-magazine training. In addition to the basic acquisition procedure, an incrementing fixed-ratio (FR) schedule was also instituted such that after every 50 reinforced responses, the FR was increased by 1 up to a final value of FR 5. Four different control conditions were used. For three, the role of the contingency between nose poking and reinforcement was investigated, and for the

Requests for reprints should be addressed to C. W. Schindler, Behavioral Pharmacology and Genetics Section, NIDA Addiction Research Center, P.O. Box 5180, Baltimore, MD 21224. fourth, levers were substituted for the nose-poke operanda to directly compare the two responses. Finally, both delay of reinforcement and deprivation level were manipulated in order to determine the influence of these variables on the acquisition of the nose-poke operant response.

\section{METHOD}

\section{Subjects}

The subjects were 75 male Long-Evans hooded rats, maintained at $80 \%$ of their free-feeding weights (approximately $400 \mathrm{~g}$ ), unless otherwise noted. They were housed individually and had free access to water.

\section{Apparatus}

The subjects were trained in four identical operant conditioning chambers (Model E10-10, Coulbourn Instruments, Allentown, PA). The chambers measured $30 \times 24 \times 29 \mathrm{~cm}$. Centered on the front wall, $3.0 \mathrm{~cm}$ above the grid floor, was a food trough (Model RPC-001, BRS/LVE, Laurel, MD) extending $1.5 \mathrm{~cm}$ into the cage and into which $45-\mathrm{mg}$ food pellets (BioServe 0021, Frenchtown, NJ) could be delivered. The nosepoke operandum (Model E21-09, Coulbourn) consisted of a 3-cm-diam hole, which registered a response via a photocell that was activated whenever a rat placed its nose into the hole at least $.5 \mathrm{~cm}$. The hole could be illuminated from behind by two yellow light-emitting diodes. Two nose-poke operanda were used, with the hole openings centered $9 \mathrm{~cm}$ on either side of the food trough and $3 \mathrm{~cm}$ above the grid floor. A houselight was centered at the top of the front wall. Directly below the houselight was a Sonalert, which was used as feedback for the nose pokes $(10-\mathrm{msec}$ pulse). When a leverpress response was tested, standard rat levers (Model E21-03, Coulbourn) replaced the nose-poke operanda at the same height from the grid floor. The entire experimental chamber was enclosed in a ventilated acoustical chamber (Model E10-20, Coulbourn). All experimental events and data collection were performed using a MED-PC computer system (Med Associates, East Fairfield, VT).
Procedures
Acquisition procedure. Two groups of subjects $(n=7)$ were trained on the incrementing fixed-ratio (FR) acquisition training procedure. One of these groups was trained independently (Group 1) and the other was used for the yoked controls described below (Group 2). During this pro- cedure, the houselight and hole lights were illuminated when the rats were placed in the training chamber. A variable-time (VT) 60-sec sched- ule was also operative such that, on average, every $60 \mathrm{sec}$ a food pellet was delivered and the houselight and hole lights were turned off for $2 \mathrm{sec}$. For each rat, one hole (either right or left, counterbalanced within groups) was defined as correct. Any nose-poke response to that hole 
was immediately followed by a food pellet and the houselight and hole lights were turned off for $2 \mathrm{sec}$. Any correct response also reset the VT to the next scheduled interval. A response to the other hole (incorrect) produced only the feedback tone. After each consecutive 50 reinforcements for correct responses, the FR was increased by 1 until an FR 5 was reached. Completed FRs were carried over across days and counted toward the completion of the next 50 reinforcements for incrementing the FR. Sessions were $30 \mathrm{~min}$ in duration and were conducted daily for five consecutive days (Monday-Friday).

Control procedure. Four separate groups of rats were used for controls. For one group $(n=8)$, the VT schedule was operative, as described above, but nose-poke responses produced only the feedback tone and were not reinforced (Group VT). For the second group $(n=4)$, the VT schedule was operative, as described above, except that the hole lights and houselight were not turned off following pellet deliveries (Group VT NL). Again, no nose pokes were reinforced. Finally, a third group $(n=7)$ was yoked to one of the groups (Group 2), as described above. For this group, pellets were delivered and the houselight and hole lights were turned off whenever that rat's yoked partner received a pellet, whether it was through the VT or correct responding. For this group also, nose pokes were not reinforced. A final set of rats $(n=7)$ was trained on the same acquisition procedure described above, except that two levers replaced the nose-poke operanda as the response manipulandum.

Delay of reinforcement. Four groups of rats were trained with delay of reinforcement manipulated. One group (Group 3, $n=8,0$-sec delay) was trained with the identical acquisition procedure described above. The training procedure for the other three groups was similar, except that a delay was instituted after reinforced nose pokes. That is, after each reinforced nose poke, there was a delay until the pellet was delivered and the houselight and hole lights were turned off. The three separate groups of rats were trained at three different delays: $1 \mathrm{sec}(n=$ $7), 3 \sec (n=9)$, and $5 \sec (n=8)$. Nose pokes emitted during the delay had no scheduled consequence.

Deprivation level. Three groups of rats were trained with deprivation level manipulated. All three groups were trained under the same procedure, identical to that described for acquisition training. One group was maintained at the $80 \%$ of free-feeding weight (Group $4, n=8$ ) condition that was used with all other groups described above, one at $87.5 \%(n=8)$, and a final group at $95 \%(n=5)$.

Data analysis. All responses were recorded and time was accumulated for each FR value, exclusive of the 2 -sec period following pellet delivery. When response rates were calculated, only correct FR responses were used and the 2 -sec periods following reinforcements as well as the time for any delay of reinforcement were excluded from the analysis. Values presented are mean $\pm S E M$. Where appropriate, an analysis of variance was performed with follow-up contrasts in order to determine individual effects (Wilkinson, 1992).

\section{RESULTS}

Figure 1 presents the results for the various control conditions, along with those of Groups 1 and 2 . The top panel presents correct responses for the nose-poke and leverpress acquisition groups. The number of correct nose pokes increased rapidly over the 5 days, reaching a level of approximately 1,000 on day 5 . In general, the rats reached FR 5 by day 3 . Unlike the nose poke, leverpress responses were not acquired rapidly. By Day 5 , the average number of correct leverpresses was only around 200 . In fact, of the 8 rats trained with the lever instead of the nose poke, only 3 reached the FR 5 criterion by the end of Day 5. This slow acquisition reflects the lower initial level of responding to the lever. Compared with the average of 20 incorrect responses on Day 1 for the nose poke, there were only $10.6 \pm 3.8$ incorrect responses for the leverpress. Incorrect responses decreased over the 5 days of the experiment.
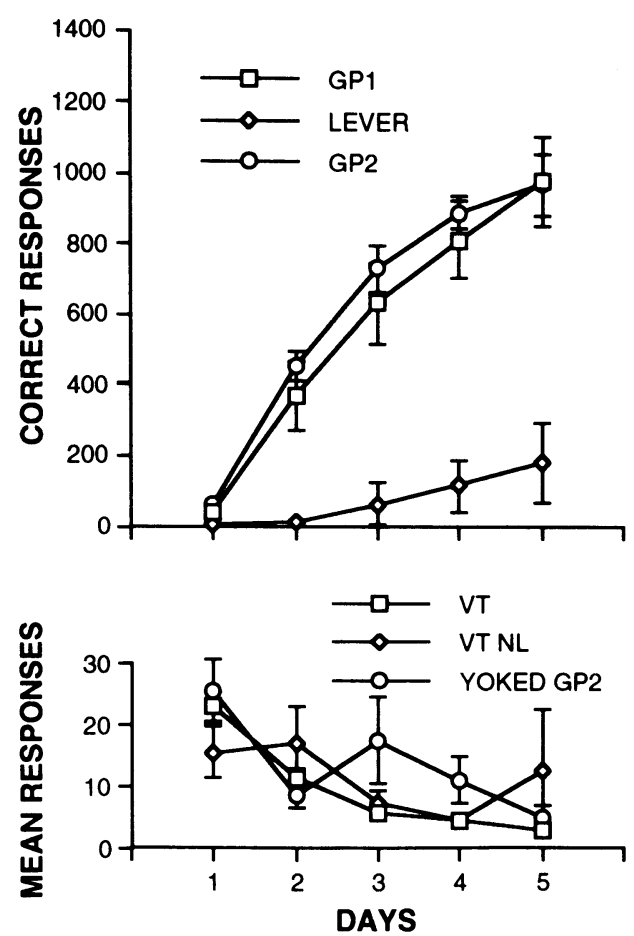

Figure 1. Comparison of nose-poke acquisition (Groups 1 and 2) with leverpress acquisition (top panel). For both nose poking and leverpressing, correct responses were reinforced with food on an FR schedule. The bottom panel shows the results of various control groups. Responses were not reinforced for any of these groups. Group VT had the VT schedule operative, as in Groups 1 and 2. For Group VT NL, the VT was active, but there was no change in the hole lights or houselight when pellets were delivered. Finally, the yoked group received the same number of pellets and the same stimulus changes with pellet delivery as did the animals in Group 2. There were 4-8 rats per group.

The bottom panel of Figure 1 presents the mean number of nose pokes for both the left and right holes for the three nose-poke control groups whose responses were not reinforced. The VT itself (Group VT) did not show any increases in responding; in fact, responding decreased over days much as it did for incorrect nose pokes for the acquisition groups. The pairing of the VT food pellets with the hole lights and houselight going off also did not affect responding, because responding for Group VT NL, for which the hole lights and houselight remained on throughout the session, was comparable to that of Group VT. Finally, the increased number of food pellets delivered to the groups of rats for which correct responding was reinforced by food presentation also did not affect acquisition independent of the reinforcement contingency. Responding for the group yoked to Group 2 was similar to the two VT groups, even though this group also received a large number of food pellets.

Delay of reinforcement slowed acquisition of responding, as shown in Figure 2. The top panel presents correct nose pokes. Because the delay itself necessarily limited the number of reinforcers that could be obtained per 30 min session, the results are also presented in terms of re- 
sponse rate in the bottom panel. Even a delay of $1 \mathrm{sec}$ was sufficient to delay acquisition. For both number of correct responses and correct response rate, on Days 4 and 5, the 1-sec delay group was significantly lower than that for the 0 -sec delay $(p<.05)$. Longer delays produced even greater decreases in responding, with both the 3- and 5-sec delay groups being significantly different from the 0 -sec delay group from Day 2 on $(p<.01)$.

As with delay of reinforcement, deprivation level clearly affected acquisition of nose poking (Figure 3 ). This was evident when deprivation was decreased from $80 \%$ to $87.5 \%$ only on the first day of training. By Day 2 , acquisition of nose poking was evident for the $87.5 \%$ group, with the level of responding being only slightly below the $80 \%$ groups $(p=.059)$. Nose poking for the $95 \%$ group, however, was lower than both the $80 \%$ and $85.5 \%$ groups throughout the 5 days of training. The initial low level of responding for the $87.5 \%$ group may reflect an overall lower level of activity for this group. Incorrect nose pokes for the $80 \%$ group on Day 1 were $19.4 \pm 4.5$, whereas they were $10.3 \pm 1.7$ and $9.4 \pm 1.4$ for the $87.5 \%$ and $90 \%$ groups, respectively.
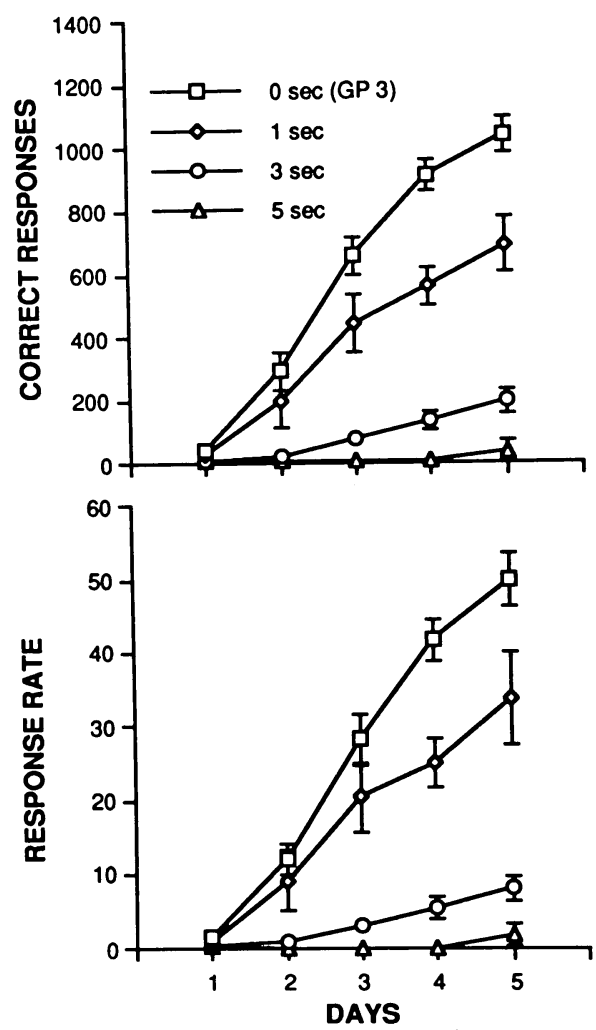

Figure 2. Effects of delay of reinforcement on acquisition of nose poking. For different groups of rats following a reinforced correct response, there was a delay of 0,3 , or 5 sec before food delivery. Responses during the delay had no consequences. The top panel presents the number of correct responses and the bottom panel presents the rate of correct responses for nose pokes $/ \mathrm{min}$. There were 7-9 rats per group.

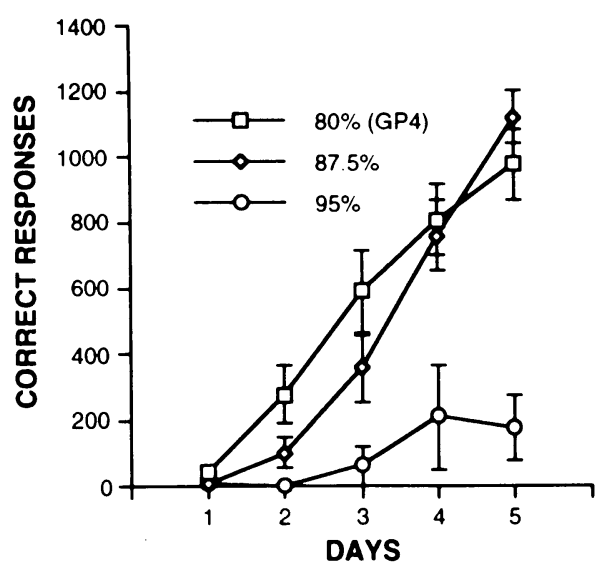

Figure 3. Effects of deprivation level on acquisition of nose poking. Different groups of rats were maintained at $80 \%, 87.5 \%$, or 95\% of their free-feeding weights. Training was identical to that shown in Figure 1. There were 5-8 rats per group.

\section{DISCUSSION}

The nose-poke operant was acquired rapidly in most rats, with close to 1,000 responses emitted per session by the fifth 30 -min session. With groups of 7-8 rats, between-group variability was low. Incorrect responses initially reflected a baseline rate of approximately 20 responses/session; however, incorrect responses decreased over days of training to a level of approximately 10 responses. This result shows the specificity of the response in terms of location; correct responses increased over days, whereas incorrect response decreased from the baseline level of operant responding.

The current data suggest that acquisition of the nose-poke response occurs much more rapidly than that for other operant responses. Although previous reports have used the nose-poke response for food in the rat, those reports either did not present detailed acquisition data (Hayes, Lattal, \& Myerson, 1979; Oakes, Rosenblum, \& Fox, 1982) or delivered the food within the hole used for the response (Davidson, Davis, \& Cook, 1971; Hayes et al., 1979), thus confounding the operant and the consumatory response. Nevertheless, these reports do confirm that the nose-poke operant is acquired rapidly. In contrast, acquisition of a leverpress response occurs slowly if there is no experimenter intervention (Lundgren \& Persinger, 1978; Miller \& Murphy, 1979; Wheatley, Welker, \& Miles, 1977). In a procedure similar to the present study, in which pellets were also presented on a VT 70-sec schedule independently of leverpressing in addition to the leverpress contingency, rats only reached a level of approximately 150 leverpresses by the eighth day of 1-h per day training sessions (Miller \& Murphy, 1979). This level of acquisition is comparable to that observed in the present study for leverpressing. It should be noted that the nose-poke response described here should not be confused with a nose-press response in which a rat is required to press its nose onto a movable plate before a response is registered (cf. Rescorla, 1992; Witkin \& Goldberg, 1990). Our own experience with this type of response (Schindler, Wu, Su, Goldberg, \& Katz, 1990) suggests that acquisition proceeds at a much slower rate with the nose press than with the nose poke.

The acquisition of the nose-poke response was clearly controlled by the contingency between the nose poke and food delivery. Without this contingency, the rate of nose poking did not increase, even when food was delivered as frequently as for the animals acquiring the response (compare Group 2 and its yoked partners). This result agrees with previous findings showing that the contingency between an operant and food is crucial for acquisition of an operant response (Davidson et al., 1971). The degree to which classical conditioning processes may have influenced response acquisition appears to be minimal. Although the 
houselight and hole lights were turned off with reinforcement, these stimulus changes did not precede reinforcement and therefore would not be expected to lead to the acquisition of conditioned responses (Mackintosh, 1974). In addition, Farwell and Ayres (1979) specifically paired hole stimuli with food presentation in a classical conditioning manner and found that, even under these conditions, operant processes appeared to predominate over classical conditioning processes in the acquisition of nose-poke responses.

The role of the delivery of response-independent food in the acquisition of nose pokes was not directly assessed. To the degree that prior magazine training can facilitate acquisition (Miller, 1976), the presentation of response-independent food may have facilitated acquisition. Previous reports on the influence of prior exposure to responseindependent food have indicated that it can either facilitate (Beatty \& Maki, 1979; Calef et al., 1984) or impair (Oakes et al., 1982) subsequent acquisition of instrumental responses. Therefore, the influence of the VT schedule, independent of magazine training, would appear to be minor.

Both delay of reinforcement and deprivation level affected acquisition in a manner consistent with a number of previous reports (cf. Mackintosh, 1974). Therefore, the present results provide further generality for the importance of these parameters to instrumental conditioning in general, and they suggest that the results obtained with the nose-poke response will have generality to other operant responses. In this regard, it can be noted that the nose-poke response appears to be more like the leverpress (Rosellini, 1978) than the instrumental runway task (Capaldi, 1978; Goomas, 1980) in that shorter delays are required to affect the operant responses than are required to affect the runway response.

In conclusion, the nose-poke operant is rapidly and consistently acquired in rats with food as the reinforcement. No experimenter intervention in the form of shaping was required for the response to be acquired. For most of the subjects, evidence of acquisition was apparent by the second 30-min session, and the rats reached response rates as high as 50 responses per minute by the fifth 30 -min session. As such, this response should be particularly useful for the study of acquisition of operant responses. This could include the study of basic behavioral processes as well as the influence of a variety of physiological parameters, including psychoactive drugs, aging, etc.

\section{REFERENCES}

Beatty, W. W., \& MaKI, W. S. (1979). Acquisition of instrumental responding following noncontingent reinforcement: Failure to observe "learned laziness" in rats. Bulletin of the Psychonomic Society, 13, 268-271.

Calef, R. S., Metz, R. A., Atrinson, T. L., Pellerzi, R. C., TayLOR, K. S., \& GELleR, E. S. (1984). Acquisition of running in the straight alley following experience with response-independent food. Bulletin of the Psychonomic Society, 22, 67-69.

CAPALDI, E. J. (1978). Effects of schedule and delay of reinforcement on acquisition speed. Animal Learning \& Behavior, 6, 330-334.

Davidson, A. B., DAvis, D. J., \& CoOK, L. (1971). A rapid technique for generating operant key-press behavior in rats. Journal of the Experimental Analysis of Behavior, 15, 123-127.
Farwell, B. J., \& Ayres, J. J. B. (1979). Stimulus-reinforcer and response-reinforcer relations in the control of conditioned appetitive headpoking ("'goal tracking") in rats. Learning \& Motivation, 10, 295-312.

Goomas, D. T. (1980). Delay of reinforcement: The role of extended training. Psychological Reports, 46, 411-414.

Hayes, S. C., Lattal, K. A., \& Myerson, W. A. (1979). Strength of experimentally induced phobic behavior in rats: Avoidance versus dual-component formulations. Psychological Reports, 44, 891-894.

Levine, T. E., Bornschein, R. L., \& Michaelson, A. (1977). Technique for assessing visual discrimination learning in mice. Pharmacology, Biochemistry \& Behavior, 7, 567-570.

Lundgren, J., \& Persinger, M. A. (1978). Activity and acquisition/ extinction comparisons of brown- and white-coated litter mates. Psychological Reports, 43, 779-782.

MACKINTOSH, N. J. (1974). The psychology of animal learning. London: Academic Press.

MeKARSKI, J. E. (1989). Main effects of current and pimozide on prepared and learned self-stimulation behaviors are on performance not reward. Pharmacology, Biochemistry \&. Behavior, 31, 845-853.

Miller, L. (1976). Acquisition of lever pressing by rats with and without magazine training. Psychological Record, 26, 355-359.

Miller, L., \& MURPHY, V. (1979). Acquisition of a simple operant by pigeons, rats, and monkeys without experimenter assistance. Bulletin of the Psychonomic Society, 13, 287-290.

OAKes, W. F., Rosenblum, J. L., \& Fox, P. E. (1982). "Manna from heaven": The effect of noncontingent appetitive reinforcers on learning in rats. Bulletin of the Psychonomic Society, 19, 123-126.

PeAR, J. J., \& Legris, J. A. (1987). Shaping by automated tracking of an arbitrary operant response. Journal of the Experimental Analysis of Behavior, 47, 241-247.

ResCorla, R. A. (1992). Response-independent outcome presentation can leave instrumental R-O associations intact. Animal Learning \& Behavior, 20, 104-111.

RoselLINI, R. A. (1978). Inescapable shock interferes with the acquisition of an appetitive operant. Animal Learning \& Behavior, 6, 155-159.

SChindler, C. W., Wu, X.-Z., Su, T.-S., Goldberg, S. R., \& KatZ, J. L. (1990), Enhanced sensitivity to behavioral effects of naltrexone in rats. Journal of Pharmacology \& Experimental Therapeutics, 252, 8-14.

Wheatley, K. L., Welker, R. L., \& Miles, R. C. (1977). Acquisition of barpressing in rats following experience with responseindependent food. Animal Learning \& Behavior, 5, 236-242.

WILKINSON, L. (1992). SYSTAT: Statistics, Version 5.2 Edition. Evanston, IL: SYSTAT, Inc.

WitKIN, J. M., \& GoldBERG, S. R. (1990). Effects of cocaine on locomotor activity and schedule-controlled behaviors of inbred rat strains. Pharmacology, Biochemistry \& Behavior, 37, 339-342.

(Manuscript received March 25, 1993.) 